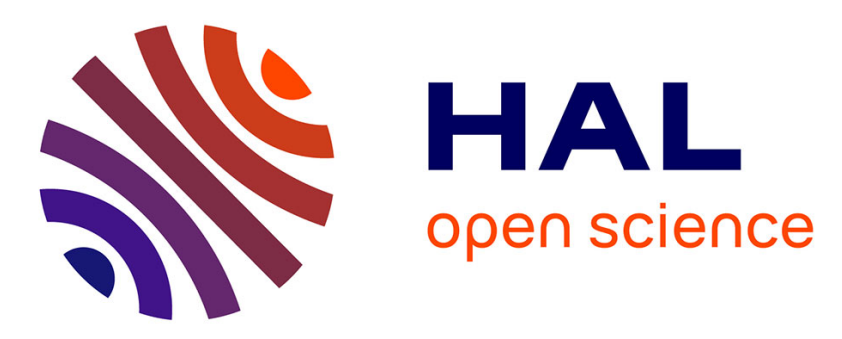

\title{
Magnetic field triggered drug release from polymersomes for cancer therapeutics
}

Hugo de Oliveira, Encarnacion Perez-Andres, Julie Thevenot, Olivier Sandre, Edurne Berra, Sébastien Lecommandoux

\section{- To cite this version:}

Hugo de Oliveira, Encarnacion Perez-Andres, Julie Thevenot, Olivier Sandre, Edurne Berra, et al.. Magnetic field triggered drug release from polymersomes for cancer therapeutics. Journal of Controlled Release, 2013, 169 (3), pp.165-170. 10.1016/j.jconrel.2013.01.013 . hal-00926568

\section{HAL Id: hal-00926568 https://hal.science/hal-00926568}

Submitted on 6 Nov 2018

HAL is a multi-disciplinary open access archive for the deposit and dissemination of scientific research documents, whether they are published or not. The documents may come from teaching and research institutions in France or abroad, or from public or private research centers.
L'archive ouverte pluridisciplinaire HAL, est destinée au dépôt et à la diffusion de documents scientifiques de niveau recherche, publiés ou non, émanant des établissements d'enseignement et de recherche français ou étrangers, des laboratoires publics ou privés. 


\title{
Magnetic field triggered drug release from polymersomes for cancer therapeutics
}

Hugo Oliveira ${ }^{\mathrm{a}, \mathrm{b}}$, Encarnación Pérez-Andrés ${ }^{\mathrm{c}}$, Julie Thevenot ${ }^{\mathrm{a}, \mathrm{b}}$, Olivier Sandre ${ }^{\mathrm{a}, \mathrm{b}}$, Edurne Berra $^{\mathrm{c}^{*}}$ and Sébastien Lecommandoux ${ }^{\mathrm{a}, \mathrm{b} *}$

a Université de Bordeaux/IPB, ENSCBP, 16 avenue Pey Berland, 33607 Pessac Cedex, France;

b CNRS, Laboratoire de Chimie des Polymères Organiques (UMR5629), Pessac, France ; c CIC bioGUNE, Parque Tecnológico de Bizkaia, 48160 Derio, Spain;

* Corresponding authors:

\section{Edurne Berra}

CIC bioGUNE, Parque Tecnológico de Bizkaia-Ed. 801A, 48160 Derio, Spain;

Tel: +34 946572506

E-mail: eberra@cicbiogune.es

\section{Sébastien Lecommandoux}

Université de Bordeaux/IPB, ENSCBP, 16 avenue Pey Berland, 33607 Pessac Cedex, France, CNRS, Laboratoire de Chimie des Polymères Organiques (UMR5629), Pessac, France;

Tel: +33 (0)540002241

E-mail: lecommandoux@enscbp.fr

\begin{abstract}
Local and temporal control of drug release has for long been a main focus in the development of novel drug carriers. Polymersomes, which can load both hydrophilic and hydrophobic species and, at the same time, be tailored to respond to a desired stimulus, have drawn much attention over the last decade. Here we describe polymersomes able to encapsulate up to $6 \%$ (w/w) of doxorubicin (DOX) together with 30\% (w/w) of superparamagnetic iron oxide nanoparticles (USPIO; $\gamma-\mathrm{Fe}_{2} \mathrm{O}_{3}$ ). Upon internalization in $\mathrm{HeLa}$ cells and when a high frequency $A C$ magnetic field $(14 \mathrm{mT}$ at $750 \mathrm{kHz})$ was applied, the developed delivery system elicited a $18 \%$ increase in cell toxicity, associated with augmented DOX release kinetics. In order to insure that the observed cytotoxicity arose from the increased doxorubicin release and not from a pure magnetic hyperthermia effect, polymersomes loaded with magnetic nanoparticles alone were also tested. In this case, no increased toxicity was observed. We
\end{abstract}


hypothesize that the magnetic field is inducing a very local hyperthermia effect at the level of the polymersomes membrane, increasing drug release. This approach opens new perspectives in the development of smart delivery systems able to release drug upon demand and therefore, improving treatment control.

\section{Keywords}

Controlled drug delivery, polymersomes, magnetic field, hyperthermia, cancer therapy, theranostic

\section{Introduction}

During the last decades a lot of efforts have been devoted to the development of more efficient cancer therapies. The nanotechnology approach has been leading the way, aiming at reduced side effects and superior outcomes. Part of this work has been focused on polymeric vesicles, also called polymersomes, which are presented as promising and versatile drug delivery systems due to their unique characteristics, i.e. compartmentalization, chemical and structure plasticity, ease to encapsulate different species, mechanical integrity and colloidal stability [1, 2]. Indeed, since they were first reported [3], extensive work has been performed regarding their preparation and physicochemical properties, leading to superior structure refinements. "Smart" polymersomes, containing stimuli-sensitive components, able to respond to internal (physio-pathological driven stimuli) and externally applied triggers have emerged [4-6], and hold promise for the development of a new generation of therapeutic tools [7]. An example of such external trigger application is the magnetically driven drug delivery, initially proposed in 1960 by Freeman et al. [8]. One can envisage a nano-delivery system that, following intravenous administration, releases its payload at the site of interest upon application of a local high frequency alternating magnetic field (HAMF).

Several inorganic magnetic cores are currently available with potential use in magnetically induced drug delivery [9]. Nevertheless, superparamagnetic iron oxide nanoparticles still constitute the better compromise between magnetic properties and biocompatibility, due to their excellent biodegradability and lack of toxicity [10]. When incorporated in polymer composite systems, they can be used to magnetically guide nanoparticles at the tumor site [11], or release a drug in a controlled manner upon deformation in a magnetic field or due to hyperthermia in a HAMF [12]. It was previously demonstrated, using a combination of mesoporous silica nanoparticles and zinc-doped iron oxide nanocrystals, loaded with doxorubicin (DOX), that exposition to a HAMF translates to an increase in cell death [13]. In 
a recent work, the team of Chen also elegantly demonstrated that capsules prepared by a double emulsion method could accommodate both anticancer drugs and magnetite nanoparticles and lead to increased cell toxicity upon internalization and exposure to a HAMF [14]. In both cases, the measured effect was due to a combination of a hyperthermic effect and the increased DOX release kinetics. The mechanism of drug controlled release was based on the so-called magnetic hyperthermia effect, where the superparamagnetic cores submitted to a HAMF produce a very local heating through dynamic hysteresis losses induced by Néel and Brownian relaxation processes [15-17]. Our group has also focused on this release strategy and developed polymersomes loaded with both maghemite ultrasmall superparamagnetic iron oxide nanoparticles (USPIO; $\gamma-\mathrm{Fe}_{2} \mathrm{O}_{3}$ ) and DOX [18]. Upon the application of a HAMF $\left(500 \mathrm{kHz}, 2.12 \mathrm{kAm}^{-1}\right)$, the drug release kinetics was significantly increased. Since no significant macroscopic temperature increase was detected, we hypothesized that a local heating, at the nanometric scale, leads to a permeation of the polymersome membrane facilitating DOX liberation. Here we demonstrate the applicability of such a system in an in cellulo scenario. In order to validate the magnetic induced intracellular delivery of DOX, HeLa cells were pre-exposed to the DOX and USPIOs loaded polymersomes and subjected to a HAMF $(750 \mathrm{KHz})$. Indeed, this HAMF was able to elicit a significant increase in cell toxicity, associated with enhanced DOX release kinetics.

\section{Material and methods}

\subsection{Materials}

Fluorescein isothiocyanate (FITC), N,N-Diisopropylethylamine (DIPEA) and anhydrous Dimethyl sulfoxide (DMSO) were purchased from Aldrich and used as received. Doxorubicin hydrochloride was purchased from Discovery Fine Chemicals (Wimborne, UK) and used as received.

Poly(trimethylene carbonate)-block-poly(L-glutamic acid) (PTMC-b-PGA) copolymer was synthesized by ring opening polymerization as previously described [19, 20]. All the experiments were conducted on a $\mathrm{PTMC}_{32}-b-\mathrm{PGA}_{14}(\mathrm{Mn}=5000 \mathrm{~g} / \mathrm{mol})$ block copolymer which presents a hydrophilic weight fraction of $35 \mathrm{wt} \%$ and a molar mass dispersity of 1.2. To allow in cellulo observations, PTMC- $b$-PGA was fluorescently labeled with fluorescein. Therefore, $250 \mathrm{mg}$ of copolymer was dissolved in $5 \mathrm{~mL}$ anhydrous DMSO and $100 \mu \mathrm{L}$ (1 eq. per acid function) DIPEA was added. After 5 min stirring, $18 \mathrm{mg}$ (1 eq.) FITC were added 
and the mixture was stirred overnight at room temperature. The resulting product (PTMC-bPGA-FITC) was dialyzed against ultrapure water for three days and freeze-dried. A labeling efficiency of $6 \mathrm{~mol} \%$ was measured by absorbance $(485 \mathrm{~nm})$.

Maghemite USPIOs $\left(\gamma-\mathrm{Fe}_{2} \mathrm{O}_{3}\right)$ were synthesized as previously described [21]. Then, USPIOs were sorted according to their size by fractionated phase separation and characterized along the sorting process by vibrating sample magnetometry (VSM) and on the final sample by scattering techniques (static and dynamic light scattering and small angle neutron scattering). Finally, they were grafted with the anionic surfactant Beycostat NB09 (CECA, Arkema group, France) as described elsewhere [22], in order to allow their inclusion in the polymersome hydrophobic reservoir. The final USPIOs presented a diameter of $9 \pm 1 \mathrm{~nm}$.

\subsection{Polymersome production}

USPIO loaded polymersomes ( $\mathrm{Np}-\mathrm{Fe})$ were obtained by the nanoprecipitation method, also named solvent assisted dispersion [23]. First, the copolymer labeling was adjusted to 0.2 mol\% by adding unlabelled PTMC- $b$-PGA; a solution in DMSO at $10 \mathrm{mg} / \mathrm{mL}$ was prepared. Hydrophobically coated USPIO nanoparticles (30\% FWR) were added to the copolymer solution. The Feed Weight Ratio (FWR) determines the ration between the mass of the engaged species (here USPIO nanoparticles) and the total copolymer mass. This solution was mixed with carbonate buffer $(50 \mathrm{mM}, \mathrm{pH} 10.5)$ using a vortex homogenizer (DMSO:buffer, 1:9 vol:vol). Nanoparticle were purified by extensive dialysis (3.5 kDa, Spectrum Labs) against phosphate buffer, $50 \mathrm{mM}$, pH 7.4.

USPIO and DOX loaded polymersomes (Np-Fe-DOX) were obtained by a similar process. In this case, DOX was included in the initial DMSO mixture at 20\% FWR. Total solid content was determined by differential weighting of the sample before and after drying for $2 \mathrm{hrs}$ at $80^{\circ} \mathrm{C}$. The total iron content was assessed by spectrophotometry $\left(\lambda_{\max }=350 \mathrm{~nm}\right)$, after dissolution of the USPIOs into ferric ions in $\mathrm{HCl} 5 \mathrm{M}$ [24]. DOX concentration was determined by spectrophotometry $\left(\lambda_{\max }=485 \mathrm{~nm}\right)$ using a DOX calibration curve. To take into account absorption of iron oxide, the absorbance of $\mathrm{Np}-\mathrm{Fe}$ of same size was first subtracted from the measured absorbance of Np-Fe-DOX and the DOX concentration was then calculated using a calibration curve in water.

Drug FWR and loading content (LC) were defined as follows:

Feed weight ratio $(\mathrm{FWR})=\frac{\text { mass engaged drug }}{\text { mass of polymer }} \times 100$ 
Loading content $(\mathrm{LC})=\frac{\text { mass encapsulated drug }}{\text { mass of polymer }} \times 100$

\section{equation 2.}

\subsection{Polymersome characterization}

Zeta potential and hydrodynamic size of the polymersomes were measured using a Zetasizer Nano ZS90 (Malvern, UK) with an automatic adjustment of attenuation and measurement time. Smoluchowski equation was applied for zeta potential determination and cumulant analysis was used for mean hydrodynamic diameter determination. All measurements were performed in triplicate, at $25^{\circ} \mathrm{C}$, in phosphate buffer $50 \mathrm{mM}$, pH 7.4.

\subsection{Cell culture}

HeLa (Human cervical carcinoma) cell line was routinely cultured in Dulbecco's Modified Eagle Medium (DMEM) with Glutamax, supplemented with $10 \%$ (v/v) foetal bovine serum (FBS). All reagents were supplied by GIBCO, Life Technologies (UK). Cells were maintained at $37^{\circ} \mathrm{C}$ in a $5 \% \mathrm{CO}_{2}$ humidified incubator. HeLa cells were plated at $15 \times 10^{3}$ cells $/ \mathrm{cm}^{2}$ for all experiments.

\subsection{Cytotoxicity assay}

To assess the viability, cells were exposed to the different formulations at designated times

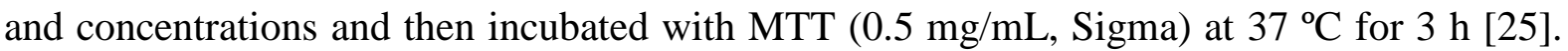
Subsequently, the medium was discarded, and the same volume of DMSO was added to dissolve the formed formazan crystals. The optical density of the supernatant was read at 540 nm using a microplate spectrophotometer (Synergy HT, Biotek, USA). Results were expressed as the percentage of the metabolic activity of treated cells relative to untreated cells.

\subsection{Cell internalization studies}

HeLa cells were sub-cultured in 24-well plates for $24 \mathrm{hrs}$ and then exposed to polymersomes at $0.10 \mathrm{mg} / \mathrm{mL}$ for 24,48 and 72 hrs. Cells were then trypsinized and analysed by flow cytometry using a FACSCanto II cytometer (BD Biosciences, USA). Twenty thousand gated events were measured for each replicate and analysed by histogram for green fluorescence using the FlowJo software (version 8.3.7, USA). For microscopy analysis, cells were exposed to polymersomes at $0.10 \mathrm{mg} / \mathrm{mL}$ for $24 \mathrm{hrs}$ and then washed with PBS, fixed with p- 
formaldehyde $3.3 \%(\mathrm{w} / \mathrm{v})$ in $\mathrm{PBS}$ for $20 \mathrm{~min}$ at $37^{\circ} \mathrm{C}$, permeabilized with TritonX-100 $0.2 \%$ $(\mathrm{w} / \mathrm{v})$ in PBS for $5 \mathrm{~min}$ at room temperature (RT). Images were acquired using the Leica TCS SP5 confocal microscope (Leica, Germany).

\subsection{Exposure to high frequency $A C$ magnetic field}

\section{$\underline{\text { Release studies: }}$}

In order to determine drug release kinetics from the developed system, $900 \mu \mathrm{L}$ of drugloaded vesicles were placed into a dialysis tube (Float-A-Lyzer, 20,000 g/mol MWCO, $1 \mathrm{~mL}$ volume, Spectra/Por) and introduced in a Falcon tube containing $50 \mathrm{~mL}$ of phosphate buffer (50 $\mathrm{mM} \mathrm{pH} \mathrm{7.4).} \mathrm{This} \mathrm{reservoir} \mathrm{was} \mathrm{immersed} \mathrm{in} \mathrm{a} \mathrm{water} \mathrm{bath} \mathrm{stabilized} \mathrm{at} 37^{\circ} \mathrm{C}$. The upper part (containing the sample in the dialysis cassette) was exposed to a HAMF for 10 minutes, 3 times a day ( 2 hours interval between exposures) during 72 hrs, by means of a 4 -turn $5.5 \mathrm{~cm}$ inductor coil (at radiofrequency of $750 \mathrm{kHz}$ and intensity of $14 \mathrm{mT}$; Seit Elettronica, Italy). At the defined sampling points, a spectrophotometric measurement at $\lambda_{\max }=485 \mathrm{~nm}$ was performed on an aliquot taken from the dialysis bag. To take into account optical absorption by iron oxide and turbidity due to the large size of the vesicles, the DOX concentration was calculated from the measured absorbance using a calibration curve in buffer after subtracting the absorbance value of similar USPIO-loaded vesicles.

\section{Temperature variation assessment:}

In order to determine if a macroscopic heating is generated by the iron oxide loaded formulations, the temperature variation was determined upon exposure to HAMF. Briefly, polymersomes were dispersed in complete culture media to a final USPIO concentration of $125 \mu \mathrm{g} / \mathrm{mL}$ (corresponding to the same polymersomes concentration used for in cellulo studies with HAMF). Medium alone was used as control with the purpose of assessing the influence of the eddy currents coming from the electrical conductivity of the high ionic strength buffer. The aforementioned media were placed in cell culture plates $(35 \mathrm{~mm}$ diameter), positioned in an adiabatic glass chamber with a double-wall were a silicone oil of low electrical and thermal conductivities (Rhodorsil $47 \mathrm{v} 100$ ) thermostated at $37^{\circ} \mathrm{C}$ was circulating. Such a chamber could fit 3 cell culture plates of $35 \mathrm{~mm}$ diameter, filled with 2.4 $\mathrm{mL}$ of DMEM supplemented with $10 \%$ serum. They were exposed to a HAMF (as previously described) for 10 minutes. Temperature was recorded every 2 seconds during 10 minutes using an optical fiber thermometer (Opsens, Québec, Canada). 


\section{In cellulo studies:}

HeLa cells were sub-cultured in $35 \mathrm{~mm}$ diameter plates during $24 \mathrm{hrs}$ and then exposed to polymersomes for another $24 \mathrm{hr}$ period. Afterwards, cell media was replaced with $2.4 \mathrm{~mL}$ of fresh media and plates were placed in the adiabatic glass chamber thermostated at $37^{\circ} \mathrm{C}$. Cells where then exposed to a HAMF, as previously described, for 10 minutes, 3 times a day (with 2 hours interval between two exposures) during 2 days. Cell viability was finally evaluated by MTT $72 \mathrm{~h}$ after the addition of the polymersomes.

\subsection{Statistical analysis}

Statistically significant differences between two groups were analysed by non-parametric Man-Whitney test, using the Graphpad Prism 5.0 software. A p value lower than 0.05 was considered statistically significant.

\section{Results and Discussion}

Hybrid polymersomes and drug loaded hybrid polymersomes were thoroughly characterized; their main properties are presented in Figure 1. The vesicular structures containing USPIOs and DOX, and fluorescently labeled with FITC present similar hydrodynamic diameters as previously described $[23,26]$. The loading of DOX did not significantly influence the size or size dispersity of polymersomes, both formulations presented a rather low polydispersity with mean hydrodynamic diameters below $200 \mathrm{~nm}$. Consistent with previous reports, no significant differences in surface charges were observed between the two formulations, with zeta potential values near $-30 \mathrm{mV}[18,23]$. Drug loaded polymersomes presented a DOX loading content (as defined by equation 2.) of $5.6 \%$, corresponding to a loading efficiency of $28 \%$. 


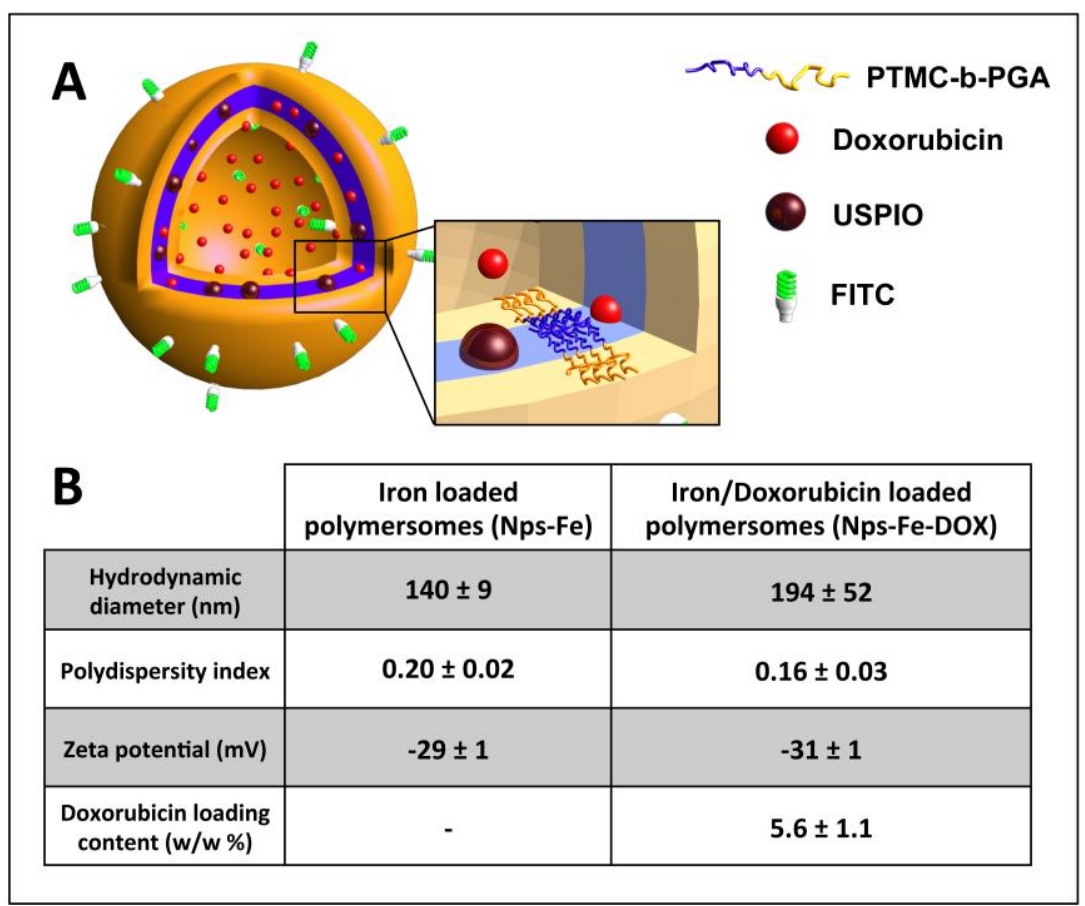

Figure 1- Schematic representation of a doxorubicin/USPIO loaded and FITC grafted polymersome (Np-Fe-DOX) structure (A). Polymersomes characterization in terms of size $(\mathrm{nm})$, polydispersity index, zeta potential $(\mathrm{mV})$ and doxorubicin loading content $(\mathrm{w} / \mathrm{w} \%)$ in phosphate buffer $(50 \mathrm{mM} \mathrm{pH} \mathrm{7.4)} \mathrm{is} \mathrm{shown} \mathrm{in} \mathrm{B} \mathrm{(Average} \pm \mathrm{SD}, \mathrm{n}=3$ ).

As means to assess the influence of the exposure of Np-Fe-DOX to a HAMF, a doxorubicin release study was performed (expressed as cumulative release, \%). As observed in figure 2, an increase of drug release was observed when the sample was exposed to the HAMF.
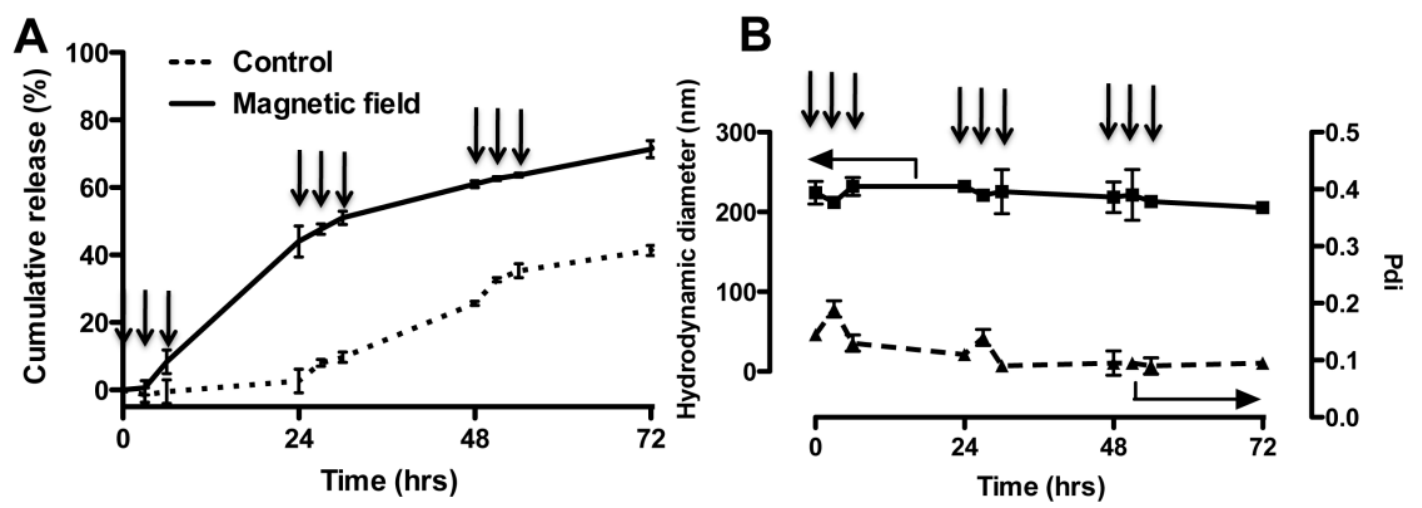

Figure 2- Cumulative drug release from USPIO and doxorubicin loaded polymersomes (NpFe-DOX) when exposed (or not) to a high frequency alternating magnetic field (indicated by the arrows) at $37^{\circ} \mathrm{C}$ in phosphate buffer $(50 \mathrm{mM}, \mathrm{pH} 7.4$; A). Polymersomes characterization in terms of size $(\mathrm{nm})$ and polydispersity index towards time, upon exposure to a high 
frequency alternating magnetic field (indicated by the arrows; B). Results represent average $\pm \mathrm{SD}, \mathrm{n}=3$.

This release rate increase is in line with work previously reported by our group [18], where similar polymeric vesicles where able to enhance their drug release by nearly two fold upon exposure to an oscillating magnetic field $(500 \mathrm{kHz})$ [18]. Additionally, and as observed in Figure $2 \mathrm{~B}$, the polymersome size and polydispersity index were maintained all along the study.

In order to determine an optimal dose for the HAMF release studies, the toxicity of polymersomes formulations was initially assessed in HeLa cells, using a wide range of concentrations (i.e. 1.2, 4, 12, $40 \mu \mathrm{g} / \mathrm{mL}$ of DOX). As expected, we observed that the encapsulation of DOX drastically decreased cell toxicity (Figure 3), supporting polymersomes stability and the slow drug release rate. A similar behaviour was previously described for other vesicular delivery systems [27, 28], where DOX encapsulation in the vesicular structures reduces the amount of available drug, what may potentially diminish unwanted off-target effects [29] and inhibit multi drug resistance [30]. Np-Fe alone did not significantly affect metabolic activity of HeLa cells at the tested conditions, although a dose response effect was observed for the 2 largest concentrations (see Figure 3).

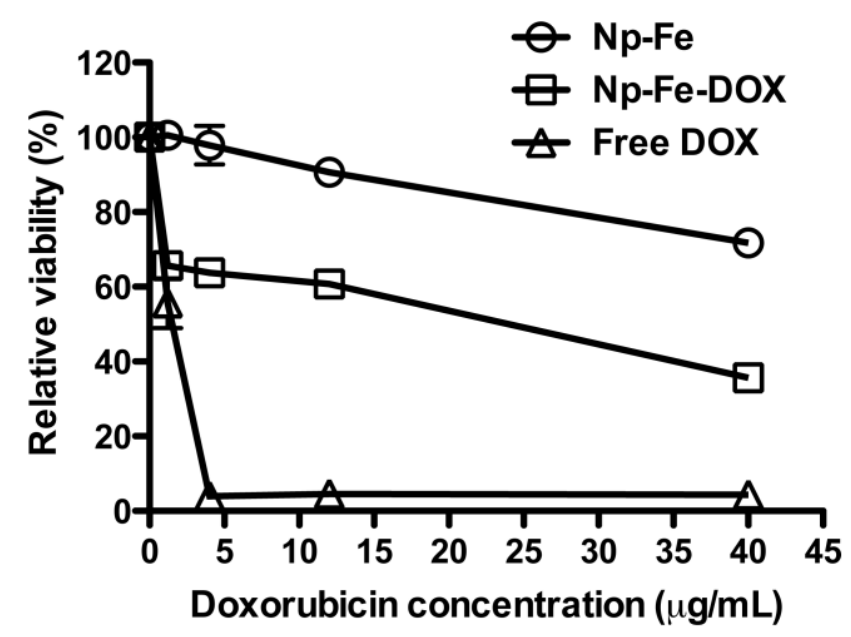

Figure 3- MTT assay of HeLa cells at $72 \mathrm{hrs}$ post exposure to USPIO loaded polymersomes (Np-Fe), USPIO and doxorubicin loaded polymersomes (Np-Fe-DOX) or free doxorubicin (Free DOX) for different doxorubicin final doses (Average $\pm \mathrm{SD}, \mathrm{n}=3$ ). 
Based on these data, the concentration of $12 \mu \mathrm{g} / \mathrm{mL}$ of DOX was established for further studies. Indeed, Np-Fe-DOX polymersomes at the selected dose shown a clear toxic effect but additional toxicity could be evaluated if, as hypothesized, HAMF induction improves drug release.

Due to the negative zeta potential and small size of the developed systems we initially expected low internalization kinetics. To confirm the efficient internalization of the developed systems by HeLa cells, uptake studies using confocal fluorescent microscopy and flow cytometry (FACS) were performed. As observed in Figure 4A, at 24 hrs post exposure, polymeric vesicles were observed in the intracellular milieu of HeLa cells (green signal). The internalization kinetics was then assessed by FACS. Figure 4B shows that the number of FITC positive cells increases with time, attaining nearly $62 \%$ of the cell population at $72 \mathrm{hrs}$ post-exposure. Nevertheless, a significant internalization was reached at $24 \mathrm{hrs}$ postexposure. This time point was used thereafter for the assessment of intracellular drug release triggered by HAMF, in order to avoid the potential polluting effect of an extracellular release of DOX.

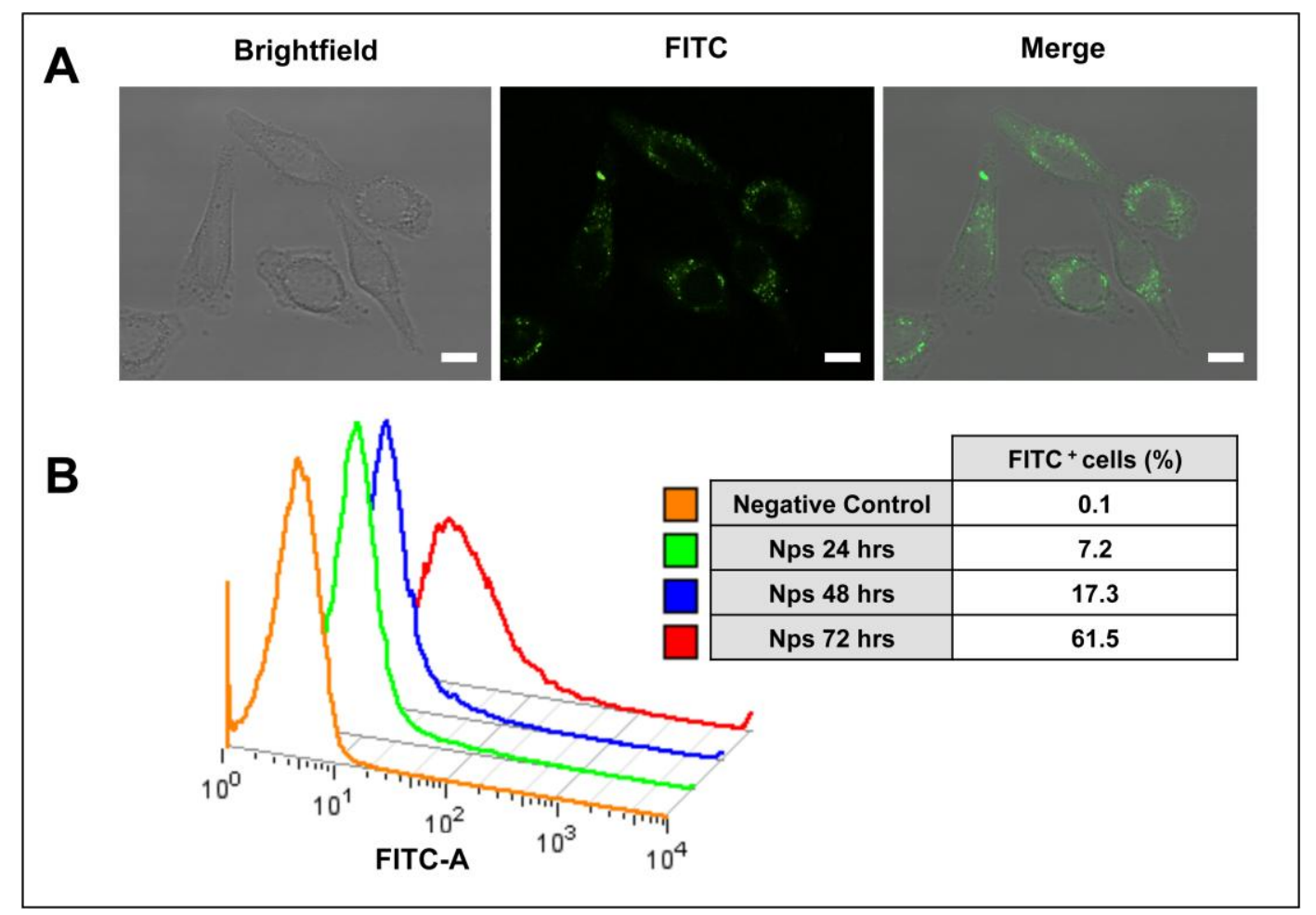

Figure 4- Internalization of USPIOs loaded polymersomes (Np-Fe) in HeLa cells by confocal fluorescence microscopy (after $24 \mathrm{hrs}$ of exposition, scale bar=10 $\mu \mathrm{m})(\mathbf{A})$ or FACS Analysis at 24, 48 and $72 \mathrm{hrs}(\mathbf{B})$. 
Since the aim of this work was focused on magneto-chemotherapy, the final USPIOs concentration used $(0.125 \mathrm{mg} / \mathrm{mL}$ of USPIOs in culture medium) was much lower than what was reported to achieve hyperthermia induced cell toxicity [31, 32]. Nevertheless, we measured the temperature increase obtained when exposing USPIOs to a HAMF in our experimental conditions. Complete cell culture media was supplemented with $\mathrm{Np}$-Fe and exposed to HAMF during 10 minutes. As observed in Figure 5, no significant temperature variation was observed as compared to media alone. The macroscopic temperature increase during the time of experiment $\left(5.4^{\circ} \mathrm{C}\right)$ being independent of the presence of the USPIOs, was then associated to an eddy current effect coming from the electrical conductivity of the high ionic strength buffer. Moreover, we have previously shown that no significant drug leakage occurred when DOX loaded polymersomes were incubated between $37^{\circ} \mathrm{C}$ and $45^{\circ} \mathrm{C}$ even for long periods of time [23]. We can therefore exclude any premature increase of DOX release due to the macroscopic heating associated with exposition to HAMF.

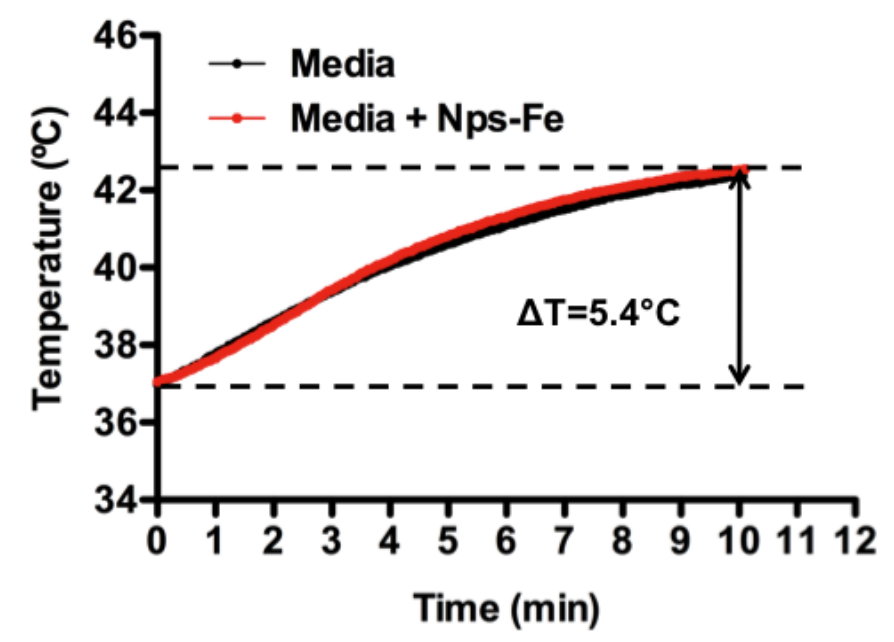

Figure 5- Temperature variation assessment of cell culture media, including (Red) or not (Black) USPIO loaded polymersomes (Np-Fe), exposed to high frequency alternating magnetic field for $10 \mathrm{~min}$.

In order to assess the effect of a HAMF on the intracellular release of DOX, cells were exposed to the polymersomes for $24 \mathrm{hrs}$ and then media was refreshed. Cells were then exposed to HAMF during the following $48 \mathrm{hrs,}$, as described in materials and methods section. As observed in Figure 6, Np-Fe with or without exposure to the magnetic field had no significant influence on cell viability. Conversely, Np-Fe-DOX exposed to HAMF induced a significant increase in cell toxicity as compared to the Np-Fe-DOX without exposure to the magnetic field. Again - and as expected - the Np-Fe-DOX exerted only a 
small fraction of the toxicity of free DOX at the same dose, indicating that DOX is released in a gradual, controlled manner.

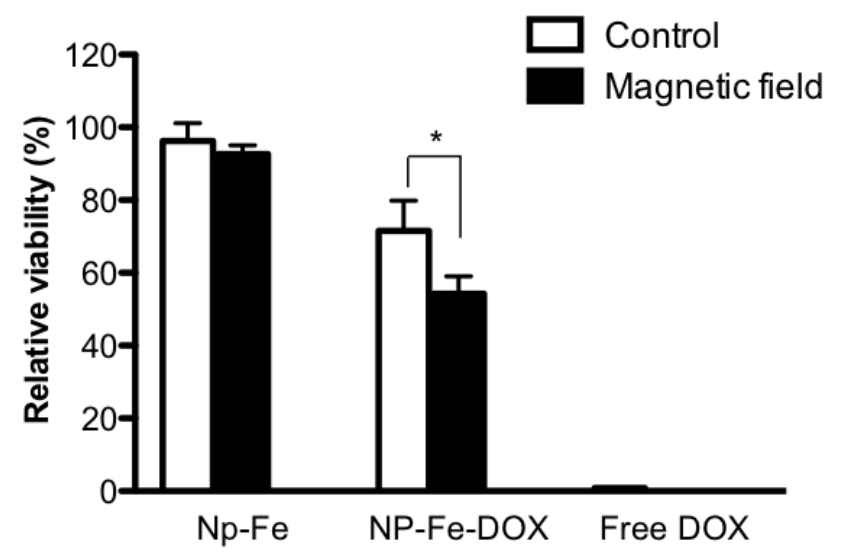

Figure 6- MTT assay of HeLa cells after $72 \mathrm{~h}$ of exposure ( $24 \mathrm{hrs}$ of incubation followed by $48 \mathrm{hrs}$ with or without exposure to the high frequency alternating magnetic field) to USPIO loaded polymersomes (Np-Fe), USPIO and doxorubicin loaded polymersomes ( $\mathrm{Np}-\mathrm{Fe}-\mathrm{DOX})$ or free doxorubicin (Free DOX) at a final dose of $12 \mu \mathrm{g} / \mathrm{mL}$ of doxorubicin (* denotes $p<0.05 ;$ Average $\pm \mathrm{SD}, \mathrm{n}=3$ ).

Taken together with the release studies (Figure 2), the results presented in Figure 6 show that upon cell internalization and exposure to a HAMF, Np-Fe-DOX are able to boost the release of DOX to the intracellular compartment and increase cell toxicity. Importantly, no cytotoxic effect was observed on control cells, exposed to the same intensity and duration of HAMF, showing that the protocol used did not induced toxicity. Indeed, as described previously, few minutes exposure to a temperature of $42{ }^{\circ} \mathrm{C}$ had minimal influence on HeLa cells viability [33].

In summary, under the influence of a high-frequency alternating magnetic field, USPIOs can generate heat through dynamic hysteresis losses induced by Néel and Brownian relaxation processes [15-17]. Although these two dissipation mechanisms contribute to the heating rate [16], it is usually believed that in the highly crowded intracellular environment, the USPIO particles being associated with intracellular compartments that impair their free rotation, the Brownian relaxation mode can be neglected [32]. We put forward that upon exposure to the oscillating magnetic field a very localized heating occurs by Néel relaxation, at the level of the polymersome structure, increasing the release rate of DOX, either by a poration effect or an increased permeability of the polymersomes membrane [18]. Indeed, and as observed in figure $2 \mathrm{~B}$, the polymersome structure maintains its integrity upon exposure to HAMF 
leading us to exclude polymersome disruption or destabilization as driving force for augmented drug delivery.

\section{Conclusions}

USPIOs have great potential for both therapeutic (e.g. hyperthermia) and diagnostic (e.g. MRI) applications; they appear especially promising for cancer treatment [34]. When immersed in the polymersome hydrophobic layer, USPIOs may serve as tools to trigger drug release when exposed to a HAMF. Additionally, one may envisage the simultaneous imaging of such carriers, in vivo, enabling unprecedented control over the therapeutic approach. The multi-compartment character of polymeric vesicles, allowing the encapsulation of both hydrophobic and hydrophilic components in separate reservoirs, enables the design of complex drug delivery systems in a simple fashion [35]. Additionally, polymersomes have shown to be able to accommodate very high levels of drug (i.e. $12 \%$ DOX LC) together with USPIOs (i.e. $50 \%$ LC) [18], as compared with other systems, like double emulsion capsules where only $2.4 \%$ (w/w) DOX LC was obtained [14]. The straightforward polymersomes preparation method is another key factor to be considered for potential scale-up of the synthesis. Additionally, due to their chemical plasticity one can envisage the grafting of targeting moieties as means to modulate their biodistribution, reducing unwanted side effects, as well as enabling them as potential diagnostic tools. Polymersomes also provide a robust and less leaky membrane than liposomes, making passive release of entrapped drugs a limitation in most cases. We used this limitation as an opportunity in order to achieve spatial and temporal control of drug release by means of an external trigger. This is, to our knowledge, the first report of an increased cytotoxic effect driven by a polymersome-based system able to increase drug release upon exposure to HAMF. This novel approach towards controlled drug delivery opens new perspectives regarding future applications in nanomedicine.

\section{Acknowledgements}

This work was supported by funding from the European Commission under the seventh framework within the frame of the NanoTher project (Integration of novel NANOparticle based technology for THERapeutics and diagnosis of different types of cancer CP-IP 213631-2). The authors would also like to thanks ESF P2M and CNRS-MI G3N ("NanoBlast”) programs for support. 


\section{Graphical abstract}

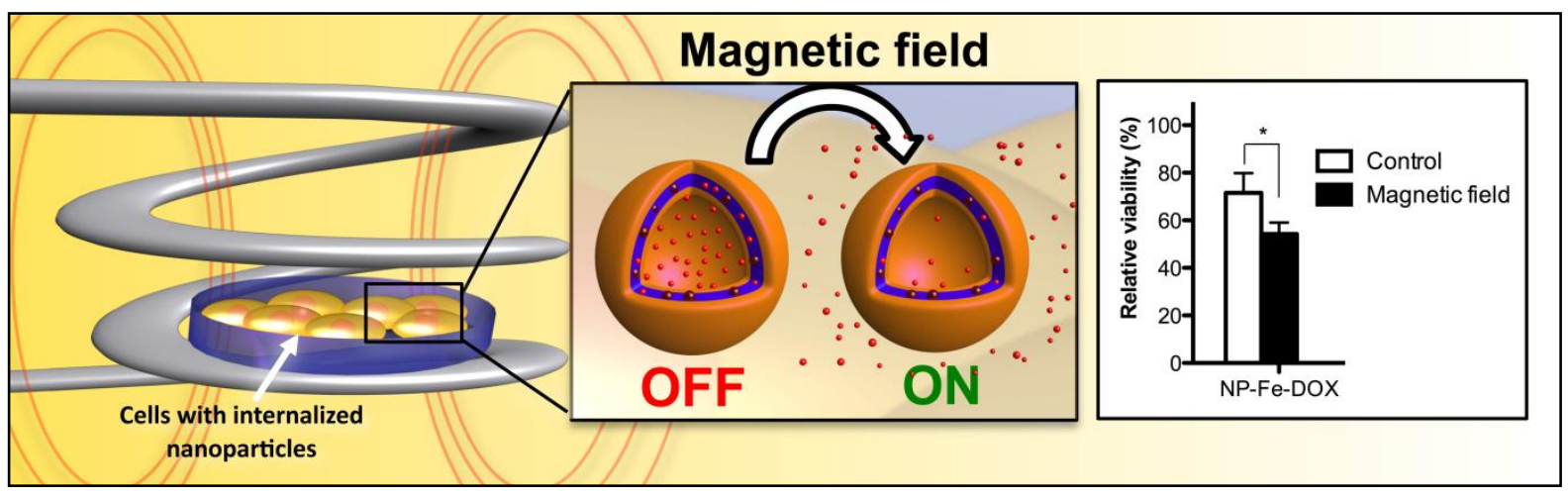

When exposed to a high frequency magnetic field, internalized hybrid polymersomes (loaded with doxorubicin and superparamagnetic iron oxide nanoparticles) are able to trigger intracellular drug release and increase cytoxicity. 


\section{References}

1. Lee JS, Feijen J. Polymersomes for drug delivery: design, formation and characterization. J Control Release. 2012;161(2):473-83.

2. Meng F, Zhong Z, Feijen J. Stimuli-responsive polymersomes for programmed drug delivery. Biomacromolecules. 2009;10(2):197-209.

3. Discher BM, Won YY, Ege DS, Lee JCM, Bates FS, Discher DE, et al. Polymersomes: Tough vesicles made from diblock copolymers. Science. 1999;284(5417):1143-6.

4. $\mathrm{Li} \mathrm{M-H,} \mathrm{Keller} \mathrm{P.} \mathrm{Stimuli-responsive} \mathrm{polymer} \mathrm{vesicles.} \mathrm{Soft} \mathrm{Matter.}$ 2009;5(5):927-37.

5. Du J, O'Reilly RK. Advances and challenges in smart and functional polymer vesicles. Soft Matter. 2009;5(19):3544-61.

6. Chen W, Meng F, Cheng R, Zhong Z. pH-Sensitive degradable polymersomes for triggered release of anticancer drugs: a comparative study with micelles. J Control Release. 2010;142(1):40-6.

7. De Oliveira H, Thevenot J, Lecommandoux S. Smart polymersomes for therapy and diagnosis: fast progress toward multifunctional biomimetic nanomedicines. Wiley Interdiscip Rev Nanomed Nanobiotechnol. 2012;4(5):525-46.

8. Freeman MW, Arrott A, Watson JHL. Magnetism in medicine. J Appl Phys. 1960(5):S404-S5.

9. Shubayev VI, Pisanic Ii TR, Jin S. Magnetic nanoparticles for theragnostics. Adv Drug Deliv Rev. 2009;61(6):467-77.

10. Bulte JWM, Kraitchman DL. Iron oxide MR contrast agents for molecular and cellular imaging. NMR in Biomedicine. 2004;17(7):484-99.

11. Arias JL, Reddy LH, Othman M, Gillet B, Desmaele D, Zouhiri F, et al. Squalene based nanocomposites: a new platform for the design of multifunctional pharmaceutical theragnostics. ACS Nano. 2011;5(2):1513-21.

12. Reddy LH, Arias JL, Nicolas J, Couvreur P. Magnetic Nanoparticles: Design and Characterization, Toxicity and Biocompatibility, Pharmaceutical and Biomedical Applications. Chem Rev. 2012;[doi: 10.1021/cr300068p].

13. Thomas CR, Ferris DP, Lee JH, Choi E, Cho MH, Kim ES, et al. Noninvasive remotecontrolled release of drug molecules in vitro using magnetic actuation of mechanized nanoparticles. J Am Chem Soc. 2010;132(31):10623-5.

14. Hu SH, Liao BJ, Chiang CS, Chen PJ, Chen IW, Chen SY. Core-shell nanocapsules stabilized by single-component polymer and nanoparticles for magnetochemotherapy/hyperthermia with multiple drugs. Adv Mater. 2012;24(27):3627-32.

15. Jordan A, Wust P, Fahling H, John W, Hinz A, Felix R. Inductive heating of ferrimagnetic particles and magnetic fluids: Physical evaluation of their potential for hyperthermia. Int J Hyperthermia 2009;25(7):499-511.

16. Rosensweig RE. Heating magnetic fluid with alternating magnetic field. J Magn Magn Mater. 2002;252(0):370-4.

17. Fortin J-P, Wilhelm C, Servais J, Menager C, Bacri J-C, Gazeau F. Size-Sorted Anionic Iron Oxide Nanomagnets as Colloidal Mediators for Magnetic Hyperthermia. J Am Chem Soc. 2007;129(9):2628-35.

18. Sanson C, Diou O, Thévenot J, Ibarboure E, Soum A, Brûlet A, et al. Doxorubicin loaded magnetic polymersomes: Theranostic nanocarriers for MR imaging and magneto-chemotherapy. ACS Nano. 2011;5(2):1122-40.

19. Le Hellaye M, Fortin N, Guilloteau J, Soum A, Lecommandoux S, Guillaume SM. Biodegradable polycarbonate-b-polypeptide and polyester-b-polypeptide block 
copolymers: synthesis and nanoparticle formation towards biomaterials. Biomacromolecules. 2008;9(7):1924-33.

20.

, Soum A, Lecommandoux Sb.

Biocompatible and Biodegradable Poly(trimethylene carbonate)-b-Poly(l-glutamic acid) Polymersomes: Size Control and Stability. Langmuir. 2009;26(4):2751-60.

21. Massart R, Dubois E, Cabuil V, Hasmonay E. Preparation and properties of monodisperse magnetic fluids. J Magn Magn Mater. 1995;149(1):1-5.

22. Da Cruz C, Sandre 0, Cabuil V. Phase behavior of nanoparticles in a thermotropic liquid crystal. J Phys Chem B. 2005;109(30):14292-9.

23. Sanson C, Schatz C, Le Meins JF, Soum A, Thévenot J, Garanger E, et al. A simple method to achieve high doxorubicin loading in biodegradable polymersomes. J Control Release. 2010;147(3):428-35.

24. Rad AM, Janic B, Iskander AS, Soltanian-Zadeh H, Arbab AS. Measurement of quantity of iron in magnetically labeled cells: comparison among different UV/VIS spectrometric methods. Biotechniques. 2007;43(5):627-8, 30, 32.

25. Mosmann T. Rapid colorimetric assay for cellular growth and survival: application to proliferation and cytotoxicity assays. J Immunol Methods. 1983;65(12):55-63.

26. Sanson C, Le Meins JF, Schatz C, Soum A, Lecommandoux S. Temperature responsive poly(trimethylene carbonate)-block-poly(l-glutamic acid) copolymer: Polymersomes fusion and fission. Soft Matter. 2010;6(8):1722-30.

27. Yang XQ, Grailer JJ, Rowland IJ, Javadi A, Hurley SA, Matson VZ, et al. Multifunctional Stable and pH-Responsive Polymer Vesicles Formed by Heterofunctional Triblock Copolymer for Targeted Anticancer Drug Delivery and Ultrasensitive MR Imaging. ACS Nano. 2010;4(11):6805-17.

28. Yang XQ, Grailer JJ, Rowland IJ, Javadi A, Hurley SA, Steeber DA, et al. Multifunctional SPIO/DOX-loaded wormlike polymer vesicles for cancer therapy and MR imaging. Biomaterials. 2010;31(34):9065-73.

29. Olson RD, Mushlin PS, Brenner DE, Fleischer S, Cusack BJ, Chang BK, et al. Doxorubicin cardiotoxicity may be caused by its metabolite, doxorubicinol. Proc Nat Acad Sci USA. 1988;85(10):3585-9.

30. Szakacs G, Paterson JK, Ludwig JA, Booth-Genthe C, Gottesman MM. Targeting multidrug resistance in cancer. Nat Rev Drug Discov. 2006;5(3):219-34.

31. Rodriguez-Luccioni HL, Latorre-Esteves M, Mendez-Vega J, Soto O, Rodriguez AR, Rinaldi C, et al. Enhanced reduction in cell viability by hyperthermia induced by magnetic nanoparticles. Int J Nanomedicine. 2011;6:373-80.

32. Fortin J-P, Gazeau F, Wilhelm C. Intracellular heating of living cells through Néel relaxation of magnetic nanoparticles. Eur Biophys J. 2008;37(2):223-8.

33. Gerner EW, Boone R, Connor WG, Hicks JA, Boone MLM. A Transient Thermotolerant Survival Response Produced by Single Thermal Doses in HeLa Cells. Cancer Research. 1976;36(3):1035-40.

34. Sailor MJ, Park JH. Hybrid nanoparticles for detection and treatment of cancer. Adv Mater. 2012;24(28):3779-802.

35. Marguet M, Bonduelle C, Lecommandoux S. Multicompartmentalized polymeric systems: towards biomimetic cellular structure and function. Chem Soc Rev. 2012; [doi:10.1039/C2CS35312A]. 